\title{
INEQUALITIES FOR THE BETA FUNCTION OF $n$ VARIABLES
}

\author{
HORST ALZER ${ }^{1}$
}

(Received 9 April, 2001)

\begin{abstract}
We present various inequalities for Euler's beta function of $n$ variables. One of our theorems states that the inequalities

$$
a_{n} \leq \frac{1}{\prod_{i=1}^{n} x_{i}}-B\left(x_{1}, \ldots, x_{n}\right) \leq b_{n}
$$

hold for all $x_{i} \geq 1(i=1, \ldots, n ; n \geq 3)$ with the best possible constants $a_{n}=0$ and $b_{n}=1-1 /(n-1)$ !. This extends a recently published result of Dragomir et al., who investigated $(*)$ for the special case $n=2$.
\end{abstract}

\section{Introduction}

The classical beta function, which is also known as Euler's integral of the first kind, is defined for positive real numbers $x$ and $y$ by

$$
B(x, y)=\int_{0}^{1} t^{x-1}(1-t)^{y-1} d t .
$$

The beta function plays a central role in the theory of special functions and also has applications in other fields, such as mathematical physics and probability theory; see $[4,5,8]$. An extension of (1.1) to $n$ variables is given by

$$
B\left(x_{1}, \ldots, x_{n}\right)=\int_{\Delta_{n-1}}\left(\prod_{i=1}^{n-1} t_{i}^{x_{i}-1}\right)\left(1-\sum_{i=1}^{n-1} t_{i}\right)^{x_{n}-1} d t_{1} \cdots d t_{n-1}
$$

$\left(x_{i}>0 ; i=1, \ldots, n ; n \geq 2\right)$, where

$$
\Delta_{n-1}=\left\{\left(t_{1}, \ldots, t_{n-1}\right) \in \mathbf{R}^{n-1} \mid t_{1} \geq 0, \ldots, t_{n-1} \geq 0, t_{1}+\cdots+t_{n-1} \leq 1\right\}
$$

${ }^{1}$ Morsbacher Str. 10, 51545 Waldbröl, Germany; e-mail: alzerhorst@freenet.de.

(C) Australian Mathematical Society 2003, Serial-fee code 1446-1811/03 
denotes the standard simplex in $\mathbf{R}^{n-1}$. There exists a close connection between $B\left(x_{1}, \ldots, x_{n}\right)$ and the gamma function,

as the elegant identity

$$
\Gamma(x)=\int_{0}^{\infty} e^{-t} t^{x-1} d t \quad(x>0)
$$

$$
B\left(x_{1}, \ldots, x_{n}\right)=\frac{\Gamma\left(x_{1}\right) \cdots \Gamma\left(x_{n}\right)}{\Gamma\left(x_{1}+\cdots+x_{n}\right)}
$$

reveals. A collection of the most important properties of the beta function of two and more variables is given, for instance, in $[4,8]$.

Various inequalities for $B(x, y)$ and $B_{p}(x, y)=\int_{0}^{p} t^{x-1}(1-t)^{y-1} d t$ appear in the literature (see $[12,13,15,16,19]$ ), whereas inequalities for the beta function of three or more variables are difficult to find. The following interesting inequality for $B(x, y)$ was published in 2000 by Dragomir et al. [9]:

$$
0 \leq 1 /(x y)-B(x, y) \leq 1 / 4 \quad(x, y \geq 1)
$$

The lower bound 0 is sharp, but the upper bound $1 / 4$ can be improved. In [3] it is shown that the second inequality of (1.2) is valid with the best possible constant $0.08731 \ldots$. It is natural to look for an extension of (1.2) to more than two variables. In this paper we determine the best possible constants $a_{n}$ and $b_{n}$ such that the doubleinequality ( $*$ ) holds for all $x_{i} \geq 1(i=1, \ldots, n ; n \geq 3)$. Furthermore, we establish several new inequalities for $B\left(x_{1}, \ldots, x_{n}\right)$, which are valid for all $n \geq 2$. In Section 3 we provide sharp constants $\alpha_{n}(c)$ and $\beta_{n}(c)$ in

$$
\alpha_{n}(c) \frac{\prod_{i=1}^{n} x_{i}^{-1 / 2+x_{i}}}{\left(\sum_{i=1}^{n} x_{i}\right)^{-1 / 2+\sum_{i=1}^{n} x_{i}}} \leq B\left(x_{1}, \ldots, x_{n}\right) \leq \beta_{n}(c) \frac{\prod_{i=1}^{n} x_{i}^{-1 / 2+x_{i}}}{\left(\sum_{i=1}^{n} x_{i}\right)^{-1 / 2+\sum_{i=1}^{n} x_{i}}}
$$

where $x_{i} \geq c>0(i=1, \ldots, n)$. Moreover, we determine the best possible upper and lower bounds for the ratio $B\left(\mu x_{1}, \mu x_{2}, \ldots, \mu x_{n}\right) / B\left(v x_{1}, v x_{2}, \ldots, v x_{n}\right)$, depending only on $\mu, v$ and $n$, and we establish that the inequalities

$$
B\left(\left(x_{1}+x_{2}\right) / 2, \ldots,\left(x_{n}+y_{n}\right) / 2\right) \leq \sqrt{B\left(x_{1}, \ldots, x_{n}\right) B\left(y_{1}, \ldots, y_{n}\right)}
$$

and

$$
B\left(x_{1}+y_{1}, \ldots, x_{n}+y_{n}\right)<\frac{1}{2^{n}}\left(B\left(x_{1}, \ldots, x_{n}\right)+B\left(y_{1}, \ldots, y_{n}\right)\right)
$$

are valid for all $x_{i}>0(i=1, \ldots, n)$. In order to prove our results we need some lemmas, which we present in the next section. 


\section{Lemmas}

First, we collect a few basic properties of the gamma function and its logarithmic derivative $\psi=\Gamma^{\prime} / \Gamma$, which is known as the psi or digamma function.

LEMMA 2.1. Let $a>0, b \geq 0$ and $x>0$ be real numbers and let $n \geq 1$ be an integer. Then we have

$$
\begin{gathered}
\Gamma(a x+b) \sim \sqrt{2 \pi} e^{-a x}(a x)^{a x+b-1 / 2} \quad(x \rightarrow \infty), \\
\log \Gamma(x) \sim(x-1 / 2) \log x-x+\frac{1}{2} \log (2 \pi)+\frac{1}{12 x}-\cdots \quad(x \rightarrow \infty), \\
\Gamma(2 x)=\frac{1}{2 \sqrt{\pi}} 4^{x} \Gamma(x) \Gamma(x+1 / 2), \\
\lim _{x \rightarrow \infty} x^{b-a} \frac{\Gamma(x+a)}{\Gamma(x+b)}=1, \\
\psi(x+1)=\psi(x)+1 / x, \\
\psi(x) \sim \log x-\frac{1}{2 x}-\frac{1}{12 x^{2}}+\frac{1}{120 x^{4}}-\cdots \quad(x \rightarrow \infty), \\
\psi^{(n)}(x)=(-1)^{n+1} \int_{0}^{\infty} e^{-x t} \frac{t^{n}}{1-e^{-t}} d t=(-1)^{n+1} n ! \sum_{k=0}^{\infty} \frac{1}{(x+k)^{n+1}}, \\
\frac{1}{x}+\frac{1}{2 x^{2}}<\psi^{\prime}(x)<\frac{1}{x}+\frac{1}{2 x^{2}}+\frac{1}{6 x^{3}} .
\end{gathered}
$$

The formulas (2.1)-(2.7) can be found in [1], while (2.8) and corresponding rational bounds for $\psi^{(n)}$ with $n \geq 2$ are given in $[2,10]$. The following two lemmas present inequalities for the psi function.

LEMMA 2.2. Let $t \geq 3$ be a real number and let $a=1-1 / \Gamma(t)$. Then we have for all real numbers $x \geq 1$ :

$$
0<a x^{t-1}+\left(a x^{t}-1\right) \psi(t x) .
$$

PROOF. We denote the expression on the right-hand side of (2.9) by $f(x)$. Differentiation gives

$$
x f^{\prime}(x)=g(x)+a(t-1) x^{t-1}+a t x^{t+1} \psi^{\prime}(t x),
$$

where $g(x)=a t x^{\prime} \psi(t x)-t x \psi^{\prime}(t x)$. Since $\psi$ is positive on $(1.461 \ldots, \infty), x \geq 1$ and $a \geq 1 / 2$ imply $g(x) /(t x) \geq \psi(t x) / 2-\psi^{\prime}(t x)$. Since $\psi$ and $-\psi^{\prime}$ are strictly increasing on $(0, \infty)$, we obtain

$$
\frac{g(x)}{t x} \geq \frac{1}{2} \psi(3)-\psi^{\prime}(3)=0.066 \ldots
$$


From (2.10) and (2.11) we conclude that $f^{\prime}(x)>0$ for $x \geq 1$. Hence we have

$$
f(x) \geq f(1)=a+(a-1) \psi(t)=\frac{h(t)}{\Gamma(t)},
$$

where $h(t)=\Gamma(t)-\psi(t)-1$. Differentiation yields $h^{\prime}(t)=\Gamma^{\prime}(t)-\psi^{\prime}(t)$ and $h^{\prime \prime}(t)=\Gamma^{\prime \prime}(t)-\psi^{\prime \prime}(t)$. Since $\Gamma^{\prime \prime}$ and $-\psi^{\prime \prime}$ are positive on $(0, \infty)$, we obtain for $t \geq 3: h^{\prime}(t) \geq h^{\prime}(3)=1.450 \ldots$ and $h(t) \geq h(3)=0.077 \ldots$, so that $(2.12)$ implies that $f$ is positive on $[1, \infty)$.

LEMMA 2.3. Let $n \geq 3$ be an integer and let $a=1-1 / \Gamma(n)$. Then we have for all real numbers $x_{i} \geq 1(i=1, \ldots, n)$ :

$$
0<\psi\left(\sum_{i=1}^{n} x_{i}\right)\left[a \prod_{i=1}^{n} x_{i}-1\right]+a\left(\max _{1 \leq i \leq n} x_{i}\right)^{-1} \prod_{i=1}^{n} x_{i} .
$$

PROOF. We may assume that $x_{1} \geq \cdots \geq x_{n} \geq 1$. Let

$$
f\left(x_{1}, \ldots, x_{n}\right)=\psi\left(\sum_{i=1}^{n} x_{i}\right)\left[a \prod_{i=1}^{n} x_{i}-1\right]+a \prod_{i=2}^{n} x_{i}
$$

and $f_{q}(x)=f\left(x, \ldots, x, x_{q+1}, \ldots, x_{n}\right)$, where $x>0$ and $q \in\{1, \ldots, n-1\}$. We prove that $f_{q}$ is increasing on $\left[x_{q+1}, \infty\right)$. Let $x \geq x_{q+1}$ and $y=q x+\sum_{i=q+1}^{n} x_{i} \geq n$. Differentiation gives

$$
\frac{1}{q} f_{q}^{\prime}(x)=\psi^{\prime}(y)\left[a x^{q} \prod_{i=q+1}^{n} x_{i}-1\right]+\psi(y) a x^{q-1} \prod_{i=q+1}^{n} x_{i}+a(1-1 / q) x^{q-2} \prod_{i=q+1}^{n} x_{i} .
$$

Since $x^{q} \prod_{i=q+1}^{n} x_{i} \geq 1, a>0, \psi(y)>0$, and $\psi^{\prime}(y)>0$, we obtain

$$
\frac{1}{q} f_{q}^{\prime}(x) \geq(a-1) \psi^{\prime}(y)+a \psi(y)=g(y), \quad \text { say. }
$$

The functions $(a-1) \psi^{\prime}$ and $a \psi$ are strictly increasing on $(0, \infty)$, so that we get

$$
\Gamma(n) g(y) \geq \Gamma(n) g(n)=\psi(n)[\Gamma(n)-1]-\psi^{\prime}(n)=h(n), \quad \text { say. }
$$

Since $h^{\prime}(n)=\psi^{\prime}(n)[\Gamma(n)-1]+\psi(n) \Gamma^{\prime}(n)-\psi^{\prime \prime}(n)>0$ for $n \geq 3$, we obtain

$$
h(n) \geq h(3)=0.527 \ldots \text {. }
$$

This implies that $f_{q}^{\prime}(x)>0$ for $x \geq x_{q+1}$. Thus we get

$$
\begin{aligned}
f\left(x_{1}, \ldots, x_{n}\right)=f_{1}\left(x_{1}\right) \geq f_{1}\left(x_{2}\right) & =f_{2}\left(x_{2}\right) \geq f_{2}\left(x_{3}\right) \geq \cdots \geq f_{n-1}\left(x_{n}\right) \\
& =a x_{n}^{n-1}+\left(a x_{n}^{n}-1\right) \psi\left(n x_{n}\right) .
\end{aligned}
$$

Applying Lemma 2.2 we conclude that $f\left(x_{1}, \ldots, x_{n}\right)>0$. 
Further, we need the following monotonicity theorem.

LEMMA 2.4. Let $a>1$ be a real number. The function

$$
\phi_{a}(x)=a(x-1 / 2) \log x-(a x-1 / 2) \log (a x)-a \log \Gamma(x)+\log \Gamma(a x)
$$

is strictly increasing on $(0, \infty)$ with $\lim _{x \rightarrow \infty} \phi_{a}(x)=-\frac{1}{2}(a-1) \log (2 \pi)$.

Proof. Let $x>0$. Differentiation gives

$$
x \phi_{a}^{\prime}(x)=-\frac{a-1}{2}-a x \log a-a x \psi(x)+a x \psi(a x)=p_{a}(x), \quad \text { say. }
$$

Further, we get

$$
\frac{1}{a} p_{a}^{\prime}(x)=-\log a-\psi(x)+\psi(a x)-x \psi^{\prime}(x)+a x \psi^{\prime}(a x)
$$

and

$$
\frac{x}{a} p_{a}^{\prime \prime}(x)=q(a x)-q(x)
$$

where $q(x)=2 x \psi^{\prime}(x)+x^{2} \psi^{\prime \prime}(x)$. Next, we prove that $q$ is strictly increasing on $(0, \infty)$. We obtain

$$
\frac{1}{x^{2}} q^{\prime}(x)=\frac{2}{x^{2}} \psi^{\prime}(x)+\frac{4}{x} \psi^{\prime \prime}(x)+\psi^{\prime \prime \prime}(x)
$$

Using the integral formulas (2.7) and

$$
\frac{1}{x^{n}}=\frac{1}{(n-1) !} \int_{0}^{\infty} e^{-x t} t^{n-1} d t \quad(x>0 ; n=1,2, \ldots),
$$

and the convolution theorem for Laplace transforms, we get

$$
\frac{1}{x^{2}} q^{\prime}(x)=\int_{0}^{\infty} e^{-x t} \Lambda(t) d t
$$

where

$$
\Lambda(t)=2 t \int_{0}^{t} \frac{s}{1-e^{-s}} d s-6 \int_{0}^{t} \frac{s^{2}}{1-e^{-s}} d s+\frac{t^{3}}{1-e^{-t}} .
$$

Let $t>0$. Then we obtain

$$
\Lambda^{\prime}(t)=2 \int_{0}^{t} \frac{s}{1-e^{-s}} d s-\left(\frac{t}{1-e^{-t}}\right)^{2}\left[1+e^{-t}(t-1)\right]
$$


and

$$
\frac{\left(1-e^{-t}\right)^{3}}{t^{2}} e^{2 t} \Lambda^{\prime \prime}(t)=2+t+(t-2) e^{t}=\sum_{k=3}^{\infty} \frac{k-2}{k !} t^{k}>0 .
$$

Since $\Lambda(0)=\Lambda^{\prime}(0)=0$, we get $\Lambda(t)>0$ for $t>0$. From (2.17) we conclude that $q$ is strictly increasing on $(0, \infty)$, so that $(2.16)$ implies $p_{a}^{\prime \prime}(x)>0$ for $x>0$. Using the asymptotic expansion (2.6) and the limit relation $\lim _{x \rightarrow \infty} x \psi^{\prime}(x)=1$, we conclude from (2.14) and (2.15) that $\lim _{x \rightarrow \infty} p_{a}(x)=\lim _{x \rightarrow \infty} p_{a}^{\prime}(x)=0$. Thus $p_{a}$ is positive on $(0, \infty)$. From $(2.14)$ we obtain that $\phi_{a}$ is strictly increasing on $(0, \infty)$.

The asymptotic formula (2.2) implies $\lim _{x \rightarrow \infty} \phi_{a}(x)=-\frac{1}{2}(a-1) \log (2 \pi)$.

\section{Main results}

We are now in a position to prove the inequalities for the beta function that we announced in Section 1. Our first theorem provides a generalisation of the doubleinequality (1.2).

THEOREM 3.1. Let $n \geq 3$ be an integer. Then we have for all real numbers $x_{i} \geq 1$ $(i=1, \ldots, n)$ :

$$
0<\frac{1}{\prod_{i=1}^{n} x_{i}}-B\left(x_{1}, \ldots, x_{n}\right) \leq 1-\frac{1}{(n-1) !} .
$$

Both bounds are best possible.

ProOF. The first inequality of (3.1) is equivalent to

$$
0<\log \Gamma\left(x_{1}+\cdots+x_{n}\right)-\sum_{i=1}^{n} \log \Gamma\left(x_{i}+1\right) .
$$

To prove (3.2) we may assume that $x_{1} \geq \cdots \geq x_{n} \geq 1$. We denote the right-hand side of (3.2) by $f\left(x_{1}, \ldots, x_{n}\right)$. Further, let $q \in\{1, \ldots, n-1\}, x \geq x_{q+1}$, and

$$
\begin{aligned}
f_{q}(x) & =f\left(x, \ldots, x, x_{q+1}, \ldots, x_{n}\right) \\
& =\log \Gamma\left(q x+\sum_{i=q+1}^{n} x_{i}\right)-q \log \Gamma(x+1)-\sum_{i=q+1}^{n} \log \Gamma\left(x_{i}+1\right) .
\end{aligned}
$$

Since $\psi$ is strictly increasing on $(0, \infty)$, we get

$$
\frac{1}{q} f_{q}^{\prime}(x)=\psi\left(q x+\sum_{i=q+1}^{n} x_{i}\right)-\psi(x+1)>0,
$$


so that $f_{q}$ is strictly increasing on $\left[x_{q+1}, \infty\right)$. This implies

$$
\begin{aligned}
f\left(x_{1}, \ldots, x_{n}\right) & =f_{1}\left(x_{1}\right) \geq f_{1}\left(x_{2}\right)=f_{2}\left(x_{2}\right) \geq f_{2}\left(x_{3}\right) \geq \cdots \geq f_{n-1}\left(x_{n}\right) \\
& =\log \Gamma\left(n x_{n}\right)-n \log \Gamma\left(x_{n}+1\right) .
\end{aligned}
$$

Let $g(x)=\log \Gamma(n x)-n \log \Gamma(x+1)$. Then we get for $x \geq 1$ :

$$
g^{\prime}(x) / n=\psi(n x)-\psi(x+1)>0
$$

and

$$
g(x) \geq g(1)=\log \Gamma(n) \geq \log \Gamma(3)=\log 2 .
$$

From (3.3) and (3.4) we conclude that (3.2) is valid.

Using the asymptotic formula (2.1) we obtain

$$
\lim _{x \rightarrow \infty}\left(1 / x^{n}-B(x, \ldots, x)\right)=\lim _{x \rightarrow \infty}\left(1 / x^{n}-(\Gamma(x))^{n} / \Gamma(n x)\right)=0
$$

which implies that in (3.1) the lower bound 0 cannot be replaced by a larger constant.

Let $a=1-1 /(n-1)$ !. To prove the right-hand side of (3.1) we have to show that

$$
0 \leq \Gamma\left(\sum_{i=1}^{n} x_{i}\right)\left[a \prod_{i=1}^{n} x_{i}-1\right]+\prod_{i=1}^{n} \Gamma\left(x_{i}+1\right)=u\left(x_{1}, \ldots, x_{n}\right), \quad \text { say. }
$$

Let $q \in\{1, \ldots, n-1\}, x_{1} \geq \cdots \geq x_{n} \geq 1$, and

$$
\begin{aligned}
u_{q}(x) & =u\left(x, \ldots, x, x_{q+1}, \ldots, x_{n}\right) \\
& =\Gamma\left(q x+\sum_{i=q+1}^{n} x_{i}\right)\left[a x^{q} \prod_{i=q+1}^{n} x_{i}-1\right]+(\Gamma(x+1))^{q} \prod_{i=q+1}^{n} \Gamma\left(x_{i}+1\right) .
\end{aligned}
$$

We set $y=q x+\sum_{i=q+1}^{n} x_{i}$ and apply Lemma 2.3. Then we get for $x \geq x_{q+1}$ :

$$
\begin{aligned}
(q \Gamma(y))^{-1} u_{q}^{\prime}(x)= & \psi(y)\left[a x^{q} \prod_{i=q+1}^{n} x_{i}-1\right]+a x^{q-1} \prod_{i=q+1}^{n} x_{i} \\
& +(\Gamma(x+1))^{q} \psi(x+1)(\Gamma(y))^{-1} \prod_{i=q+1}^{n} \Gamma\left(x_{i}+1\right)>0 .
\end{aligned}
$$

Hence $u_{q}$ is strictly increasing on $\left[x_{q+1}, \infty\right)$. This implies

$$
\begin{aligned}
u\left(x_{1}, \ldots, x_{n}\right) & =u_{1}\left(x_{1}\right) \geq u_{1}\left(x_{2}\right)=u_{2}\left(x_{2}\right) \geq \cdots \geq u_{n-1}\left(x_{n}\right) \\
& =\left(a x_{n}^{n}-1\right) \Gamma\left(n x_{n}\right)+\left(\Gamma\left(x_{n}+1\right)\right)^{n} .
\end{aligned}
$$


Let $v(x)=\left(a x^{n}-1\right) \Gamma(n x)+(\Gamma(x+1))^{n}$. Then we have

$$
\frac{v^{\prime}(x)}{n \Gamma(n x)}=a x^{n-1}+\left(a x^{n}-1\right) \psi(n x)+\frac{(\Gamma(x+1))^{n} \psi(x+1)}{\Gamma(n x)} .
$$

From Lemma 2.2 we conclude that $v$ is strictly increasing on $[1, \infty)$. Thus

$$
v(x) \geq v(1)=0 \text { for } x \geq 1 \text {, }
$$

so that (3.5) yields $u\left(x_{1}, \ldots, x_{n}\right) \geq 0$.

If $x_{1}=\cdots=x_{n}=1$, then the second inequality of (3.1) holds with equality. This implies that the upper bound $1-1 /(n-1)$ ! is sharp.

REMARK. The inequalities (3.1) are not valid for all positive real numbers $x_{i}$ $(i=1, \ldots, n)$. More precisely: there do not exist constants $c_{1}(n)$ and $c_{2}(n)$ such that

$$
c_{1}(n) \leq \frac{1}{\prod_{i=1}^{n} x_{i}}-B\left(x_{1}, \ldots, x_{n}\right) \leq c_{2}(n)
$$

holds for all $x_{i}>0(i=1, \ldots, n ; n \geq 2)$. Indeed, if we set $x_{1}=\cdots=x_{n-1}=x>0$ and $x_{n}=y>1$, then the left-hand side of (3.6) yields

$$
x^{n-1} y c_{1}(n) \leq 1-\frac{(\Gamma(x+1))^{n-1} \Gamma(y+1)}{\Gamma((n-1) x+y)} .
$$

We let $x$ tend to 0 and obtain the incorrect inequality $0 \leq 1-\Gamma(y+1) / \Gamma(y)=1-y$. And, if we set $x_{1}=\cdots=x_{n}=x>0$, then the right-hand side of (3.6) gives

$$
\frac{1}{x^{n}}-\frac{(\Gamma(x))^{n}}{\Gamma(n x)}=\frac{\Gamma(n x+1)-n x(\Gamma(x+1))^{n}}{x^{n} \Gamma(n x+1)} \leq c_{2}(n) .
$$

This is false, since the term on the left-hand side tends to $\infty$, if we let $x$ tend to 0 .

The next theorem provides sharp upper and lower bounds for $B\left(x_{1}, \ldots, x_{n}\right)$, which are valid in $[c, \infty)^{n}$, where $c>0$ is a fixed real number.

THEOREM 3.2. Let $c>0$ be a real number and let $n \geq 2$ be an integer. Then we have for all real numbers $x_{i} \geq c(i=1, \ldots, n)$ :

$$
\alpha_{n}(c) \frac{\prod_{i=1}^{n} x_{i}^{-1 / 2+x_{i}}}{\left(\sum_{i=1}^{n} x_{i}\right)^{-1 / 2+\sum_{i=1}^{n} x_{i}}}<B\left(x_{1}, \ldots, x_{n}\right) \leq \beta_{n}(c) \frac{\prod_{i=1}^{n} x_{i}^{-1 / 2+x_{i}}}{\left(\sum_{i=1}^{n} x_{i}\right)^{-1 / 2+\sum_{i=1}^{n} x_{i}}}
$$

with the best possible constants

$$
\alpha_{n}(c)=(2 \pi)^{(n-1) / 2} \quad \text { and } \quad \beta_{n}(c)=n^{n c-1 / 2} c^{(n-1) / 2} \frac{\left(\Gamma(c)^{n}\right.}{\Gamma(n c)} .
$$


ProOF. Let $x>0$ and $x_{i}>0(i=1, \ldots, n)$ be real numbers and let $q \in\{1, \ldots, n-1\}$. We define

$$
\begin{aligned}
f\left(x_{1}, \ldots, x_{n}\right)= & \sum_{i=1}^{n}\left(x_{i}-1 / 2\right) \log x_{i}-\left(\sum_{i=1}^{n} x_{i}-\frac{1}{2}\right) \log \left(\sum_{i=1}^{n} x_{i}\right) \\
& -\sum_{i=1}^{n} \log \Gamma\left(x_{i}\right)+\log \Gamma\left(\sum_{i=1}^{n} x_{i}\right)
\end{aligned}
$$

and

$$
\begin{aligned}
f_{q}(x)= & f\left(x, \ldots, x, x_{q+1}, \ldots, x_{n}\right) \\
= & q\left(x-\frac{1}{2}\right) \log x+\sum_{i=q+1}^{n}\left(x_{i}-1 / 2\right) \log x_{i} \\
& -\left(q x+\sum_{i=q+1}^{n} x_{i}-\frac{1}{2}\right) \log \left(q x+\sum_{i=q+1}^{n} x_{i}\right)-q \log \Gamma(x) \\
& -\sum_{i=q+1}^{n} \log \Gamma\left(x_{i}\right)+\log \Gamma\left(q x+\sum_{i=q+1}^{n} x_{i}\right) .
\end{aligned}
$$

Then we get $f_{q}^{\prime}(x) / q=g(x)-g(y)$, where $g(z)=\log z-1 /(2 z)-\psi(z)$ and $y=$ $q x+\sum_{i=q+1}^{n} x_{i}$. The left-hand side of (2.8) implies $g^{\prime}(z)=1 / z+1 /\left(2 z^{2}\right)-\psi^{\prime}(z)<0$ for $z>0$. Hence we conclude from $y>x$ that $g(y)<g(x)$. This implies that $f_{q}$ is strictly increasing on $(0, \infty)$.

To prove the right-hand inequality of (3.7) with $\beta_{n}(c)$ as defined in (3.8), we assume that $x_{1} \geq \cdots \geq x_{n} \geq c$. Then we obtain

$$
\begin{aligned}
f\left(x_{1}, \ldots, x_{n}\right) & =f_{1}\left(x_{1}\right) \geq f_{1}\left(x_{2}\right)=f_{2}\left(x_{2}\right) \geq f_{2}\left(x_{3}\right) \geq \cdots \geq f_{n-1}\left(x_{n}\right) \\
& =\phi_{n}\left(x_{n}\right),
\end{aligned}
$$

where $\phi_{n}$ is defined in (2.13). From Lemma 2.4 we get

$$
\phi_{n}\left(x_{n}\right) \geq \phi_{n}(c)=-\log \beta_{n}(c),
$$

so that (3.9) and (3.10) lead to

$$
f\left(x_{1}, \ldots, x_{n}\right) \geq-\log \beta_{n}(c),
$$

which is equivalent to the second inequality of (3.7). Moreover, since $f_{q}$ and $\phi_{n}$ are strictly monotonic, we conclude that the sign of equality holds in (3.11) if and only if $x_{1}=\cdots=x_{n}=c$.

To prove the left-hand side of (3.7) with $\alpha_{n}(c)=(2 \pi)^{(n-1) / 2}$ we suppose that $c \leq x_{1} \leq \cdots \leq x_{n}$. The monotonicity of $f_{q}$ and Lemma 2.4 lead to

$$
f\left(x_{1}, \ldots, x_{n}\right)=f_{1}\left(x_{1}\right) \leq f_{1}\left(x_{2}\right)=f_{2}\left(x_{2}\right) \leq f_{2}\left(x_{3}\right) \leq \cdots \leq f_{n-1}\left(x_{n}\right)
$$




$$
=\phi_{n}\left(x_{n}\right)<(-1 / 2)(n-1) \log (2 \pi)=-\log \alpha_{n}(c),
$$

which leads to the first inequality of (3.7) with $\alpha_{n}(c)=(2 \pi)^{(n-1) / 2}$.

Conversely, we assume that the left-hand inequality of (3.7) is valid for all $x_{i} \geq c$ $(i=1, \ldots, n)$. Then we set $x_{1}=\cdots=x_{n}=x>0$ and obtain $\alpha_{n}(c)<e^{-\phi_{n}(x)}$. Applying Lemma 2.4 we get $\alpha_{n}(c) \leq \lim _{x \rightarrow \infty} e^{-\phi_{n}(x)}=(2 \pi)^{(n-1) / 2}$. Thus in (3.7) the factor $\alpha_{n}(c)=(2 \pi)^{(n-1) / 2}$ cannot be replaced by a larger constant.

If a function $f$ satisfies the inequality $f\left(\delta x_{1}, \ldots, \delta x_{n}\right) \leq \delta f\left(x_{1}, \ldots, x_{n}\right)$ for all $x_{i}>0(i=1, \ldots, n)$ and $\delta \in(0,1)$, then $f$ is said to be starshaped on $\mathbf{R}_{+}^{n}$. Interesting properties of these functions can be found in $[6,7]$. As an immediate consequence of the following theorem we obtain that the beta function is not starshaped on $\mathbf{R}_{+}^{n}$.

THEOREM 3.3. Let $\mu$ and $\nu$ be real numbers with $\mu>\nu>0$ and let $n \geq 2$ be an integer. Then we have for all real numbers $x_{i}>0(i=1, \ldots, n)$ :

$$
0<\frac{B\left(\mu x_{1}, \mu x_{2}, \ldots, \mu x_{n}\right)}{B\left(\nu x_{1}, v x_{2}, \ldots, \nu x_{n}\right)}<\left(\frac{\nu}{\mu}\right)^{n-1} .
$$

Both bounds are best possible.

PROOF. To establish the second inequality of (3.12) it suffices to show that the function $f(t)=t^{n-1} B\left(t x_{1}, \ldots, t x_{n}\right)$ is strictly decreasing on $(0, \infty)$. Let $t>0$. Differentiation yields

$$
\frac{t}{f(t)} f^{\prime}(t)=n-1+\sum_{i=1}^{n} t x_{i} \psi\left(t x_{i}\right)-\psi\left(\sum_{i=1}^{n} t x_{i}\right) \sum_{i=1}^{n} t x_{i} .
$$

We set $y_{i}=t x_{i}>0(i=1, \ldots, n)$ and define

$$
g\left(y_{1}, \ldots, y_{n}\right)=\psi\left(\sum_{i=1}^{n} y_{i}\right) \sum_{i=1}^{n} y_{i}-\sum_{i=1}^{n} y_{i} \psi\left(y_{i}\right) .
$$

In order to prove

$$
g\left(y_{1}, \ldots, y_{n}\right)>n-1
$$

we assume that $y_{1} \geq \cdots \geq y_{n}>0$. Let $q \in\{1, \ldots, n-1\}, y>0$, and

$$
\begin{aligned}
g_{q}(y) & =g\left(y, \ldots, y, y_{q+1}, \ldots, y_{n}\right) \\
& =\psi\left(q y+\sum_{i=q+1}^{n} y_{i}\right)\left(q y+\sum_{i=q+1}^{n} y_{i}\right)-q y \psi(y)-\sum_{i=q+1}^{n} y_{i} \psi\left(y_{i}\right) .
\end{aligned}
$$


Then we get

where

$$
g_{q}^{\prime}(y) / q=h(z)-h(y)
$$

$$
h(x)=\psi(x)+x \psi^{\prime}(x) \text { and } z=q y+\sum_{i=q+1}^{n} y_{i}
$$

Using the series representation (2.7) we obtain

$$
h^{\prime}(x)=2 \psi^{\prime}(x)+x \psi^{\prime \prime}(x)=2 \sum_{k=1}^{\infty} \frac{k}{(x+k)^{3}}>0 .
$$

Since $z>y$, we get $h(z)>h(y)$, so that (3.15) implies that $g_{q}$ is strictly increasing on $(0, \infty)$. Hence we have

$$
\begin{aligned}
g\left(y_{1}, \ldots, y_{n}\right) & =g_{1}\left(y_{1}\right) \geq g_{1}\left(y_{2}\right)=g_{2}\left(y_{2}\right) \geq \cdots \geq g_{n-1}\left(y_{n}\right) \\
& =n y_{n}\left[\psi\left(n y_{n}\right)-\psi\left(y_{n}\right)\right] .
\end{aligned}
$$

Let

$$
\omega(y)=n y[\psi(n y)-\psi(y)]
$$

Then $\omega^{\prime}(y) / n=h(n y)-h(y)$, where $h$ is defined in (3.16). Since $h$ is strictly increasing on $(0, \infty)$, we obtain $\omega^{\prime}(y)>0$ and

$$
\omega(y)>\lim _{t \rightarrow 0} \omega(t) \quad(y>0) .
$$

The recurrence formula (2.5) implies

$$
\lim _{t \rightarrow 0} \omega(t)=n-1
$$

so that (3.17)-(3.20) lead to (3.14). From (3.13) and (3.14) we conclude that $f$ is strictly decreasing on $(0, \infty)$.

If we set $x_{1}=\cdots=x_{n}=x>0$, then we have

$$
\frac{B\left(\mu x_{1}, \ldots, \mu x_{n}\right)}{B\left(\nu x_{1}, \ldots, v x_{n}\right)}=\left(\frac{\Gamma(\mu x+1)}{\Gamma(\nu x+1)}\right)^{n} \frac{\Gamma(n \nu x+1)}{\Gamma(n \mu x+1)}\left(\frac{\nu}{\mu}\right)^{n-1}
$$

This implies

$$
\lim _{x \rightarrow 0} \frac{B\left(\mu x_{1}, \ldots, \mu x_{n}\right)}{B\left(v x_{1}, \ldots, v x_{n}\right)}=\left(\frac{v}{\mu}\right)^{n-1}
$$


And, if we put $x_{1}=x>0, x_{2}=\cdots=x_{n}=1$, then we get

$$
\begin{aligned}
\frac{B\left(\mu x_{1}, \ldots, \mu x_{n}\right)}{B\left(\nu x_{1}, \ldots, \nu x_{n}\right)}= & \frac{\Gamma(\mu x)}{\Gamma(\mu x+(n-1) \mu)}(\mu x)^{(n-1) \mu} \frac{\Gamma(\nu x+(n-1) \nu)}{\Gamma(\nu x)}(v x)^{(1-n) \nu} \\
& \times\left(\frac{\nu^{\nu} \Gamma(\mu)}{\mu^{\mu} \Gamma(\nu)}\right)^{n-1} x^{(n-1)(\nu-\mu)}
\end{aligned}
$$

From (2.4) and (3.22) we obtain

$$
\lim _{x \rightarrow \infty} \frac{B\left(\mu x_{1}, \ldots, \mu x_{n}\right)}{B\left(v x_{1}, \ldots, v x_{n}\right)}=0 .
$$

The limit relations (3.21) and (3.23) imply that the bounds given in (3.12) are best possible.

A function $f: \mathbf{R}_{+}^{n} \rightarrow \mathbf{R}$ is called midconvex (or Jensen-convex) if we have for all $x_{i}, y_{i}>0(i=1, \ldots, n)$ :

$$
f\left(\left(x_{1}+y_{1}\right) / 2, \ldots,\left(x_{n}+y_{n}\right) / 2\right) \leq \frac{1}{2}\left(f\left(x_{1}, \ldots, x_{n}\right)+f\left(y_{1}, \ldots, y_{n}\right)\right) .
$$

It is known that a continuous midconvex function is also convex; see [17]. We now prove that $f\left(x_{1}, \ldots, x_{n}\right)=\log B\left(x_{1}, \ldots, x_{n}\right)$ satisfies (3.24), which implies that the beta function is log-convex on $\mathbf{R}_{+}^{n}$. This extends a result given in [9], where a proof for the log-convexity of $B(x, y)$ is given.

THEOREM 3.4. Let $n \geq 2$ be an integer. Then we have for all real numbers with $x_{i}>0$ and $y_{i}>0(i=1, \ldots, n)$ :

$$
0<\frac{B\left(\left(x_{1}+y_{1}\right) / 2, \ldots,\left(x_{n}+y_{n}\right) / 2\right)}{\sqrt{B\left(x_{1}, \ldots, x_{n}\right) B\left(y_{1}, \ldots, y_{n}\right)}} \leq 1 \text {. }
$$

Both bounds are best possible.

ProOF. The Cauchy-Schwarz inequality for integrals yields

$$
\begin{aligned}
\left(B\left(x_{1}+y_{1}, x_{2}+y_{2}\right)\right)^{2} & =\left(\int_{0}^{1} t^{x_{1}-1 / 2}(1-t)^{x_{2}-1 / 2} t^{y_{1}-1 / 2}(1-t)^{y_{2}-1 / 2} d t\right)^{2} \\
& \leq \int_{0}^{1} t^{2 x_{1}-1}(1-t)^{2 x_{2}-1} d t \int_{0}^{1} t^{2 y_{1}-1}(1-t)^{2 y_{2}-1} d t \\
& =B\left(2 x_{1}, 2 x_{2}\right) B\left(2 y_{1}, 2 y_{2}\right) .
\end{aligned}
$$

Using the representation

$$
B\left(x_{1}, \ldots, x_{n}\right)=\prod_{i=1}^{n-1} B\left(\sum_{j=1}^{i} x_{j}, x_{i+1}\right)
$$


and (3.26) we obtain

$$
\begin{aligned}
\left(B\left(x_{1}+y_{1}, \ldots, x_{n}+y_{n}\right)\right)^{2} & =\prod_{i=1}^{n-1}\left[B\left(\sum_{j=1}^{i} x_{j}+\sum_{j=1}^{i} y_{j}, x_{i+1}+y_{i+1}\right)\right]^{2} \\
& \leq \prod_{i=1}^{n-1}\left[B\left(2 \sum_{j=1}^{i} x_{j}, 2 x_{i+1}\right) B\left(2 \sum_{j=1}^{i} y_{j}, 2 y_{i+1}\right)\right] \\
& =B\left(2 x_{1}, \ldots, 2 x_{n}\right) B\left(2 y_{1}, \ldots, 2 y_{n}\right)
\end{aligned}
$$

This proves the right-hand side of (3.25). If we set $x_{i}=y_{i}=z>0(i=1, \ldots, n)$, then equality holds in the second inequality of (3.25). Further, we have

$$
\lim _{x_{1} \rightarrow 0} \frac{\left(B\left(\left(x_{1}+y_{1}\right) / 2, \ldots,\left(x_{n}+y_{n}\right) / 2\right)\right)^{2}}{B\left(x_{1}, \ldots, x_{n}\right) B\left(y_{1}, \ldots, y_{n}\right)}=0,
$$

so that in (3.25) the lower bound 0 cannot be improved.

A function $f: \mathbf{R}_{+}^{n} \rightarrow \mathbf{R}$ is said to be subadditive if the inequality

$$
f\left(x_{1}+y_{1}, \ldots, x_{n}+y_{n}\right) \leq f\left(x_{1}, \ldots, x_{n}\right)+f\left(y_{1}, \ldots, y_{n}\right)
$$

holds for all $x_{i}, y_{i}>0(i=1, \ldots, n)$. Subadditive functions play a role in the theory of differential equations, in the theory of convex bodies, and also in the theory of semi-groups; see [18]. From the following theorem we conclude that for all real numbers $c>0$ the function $\left(x_{1}, \ldots, x_{n}\right) \mapsto\left(B\left(x_{1}, \ldots, x_{n}\right)\right)^{c}$ is subadditive on $\mathbf{R}_{+}^{n}$.

THEOREM 3.5. Let $c>0$ be a real number and let $n \geq 2$ be an integer. Then we have for all real numbers $x_{i}>0$ and $y_{i}>0(i=1, \ldots, n)$ :

$$
0<\frac{\left(B\left(x_{1}+y_{1}, \ldots, x_{n}+y_{n}\right)\right)^{c}}{\left(B\left(x_{1}, \ldots, x_{n}\right)\right)^{c}+\left(B\left(y_{1}, \ldots, y_{n}\right)\right)^{c}}<2^{-c(n-1)-1} .
$$

Both bounds are best possible.

ProOF. To prove the second inequality of (3.28) we apply Theorem 3.4, the arithmetic mean-geometric mean inequality, and Theorem 3.3 (with $\mu=2, \nu=1$ ). Then we get

$$
\begin{aligned}
\left(B\left(x_{1}+y_{1}, \ldots, x_{n}+y_{n}\right)\right)^{c} & \leq\left[B\left(2 x_{1}, \ldots, 2 x_{n}\right) B\left(2 y_{1}, \ldots, 2 y_{n}\right)\right]^{c / 2} \\
& \leq \frac{1}{2}\left[\left(B\left(2 x_{1}, \ldots, 2 x_{n}\right)\right)^{c}+\left(B\left(2 y_{1}, \ldots, 2 y_{n}\right)\right)^{c}\right] \\
& <2^{-c(n-1)-1}\left[\left(B\left(x_{1}, \ldots, x_{n}\right)\right)^{c}+\left(B\left(y_{1}, \ldots, y_{n}\right)\right)^{c}\right] .
\end{aligned}
$$


It remains to show that the bounds given in (3.28) are sharp. First, we set $x_{i}=y_{i}=$ $z>0(i=1, \ldots, n)$. The duplication formula (2.3) leads to, say,

$$
\frac{\left(B\left(x_{1}+y_{1}, \ldots, x_{n}+y_{n}\right)\right)^{c}}{\left(B\left(x_{1}, \ldots, x_{n}\right)\right)^{c}+\left(B\left(y_{1}, \ldots, y_{n}\right)\right)^{c}}=\frac{1}{2}\left(\frac{1}{2 \sqrt{\pi}}\right)^{c(n-1)}\left(\frac{(\Gamma(z+1 / 2))^{n}}{\Gamma(n z+1 / 2)}\right)^{c}=f(z) .
$$

Since $\Gamma(1 / 2)=\sqrt{\pi}$, we obtain

And using (2.1) we get

$$
\lim _{z \rightarrow 0} f(z)=2^{-c(n-1)-1} .
$$

$$
\lim _{z \rightarrow \infty} f(z)=0
$$

From (3.29) and (3.30) we conclude that both bounds in (3.28) are best possible.

REMARK. A multiplicative analogue of the definition (3.27) is given by

$$
f\left(x_{1} y_{1}, \ldots, x_{n} y_{n}\right) \leq f\left(x_{1}, \ldots, x_{n}\right) f\left(y_{1}, \ldots, y_{n}\right) .
$$

If $f$ satisfies (3.31) for all $x_{i}, y_{i}>0(i=1, \ldots, n)$, then $f$ is said to be submultiplicative on $\mathbf{R}_{+}^{n}$. These functions have applications in functional analysis and group theory; see [11,14]. If (3.31) holds with " $\geq$ " instead of " $\leq$ ", then $f$ is called supermultiplicative. Let $n \geq 2$. We set $x_{i}=1(2 \leq i \leq n)$ and $y_{i}=1(1 \leq i \leq n ; i \neq 2)$. Then we obtain, say,

$$
\begin{aligned}
\frac{B\left(x_{1} y_{1}, \ldots, x_{n} y_{n}\right)}{B\left(x_{1}, \ldots, x_{n}\right) B\left(y_{1}, \ldots, y_{n}\right)} & =\frac{\Gamma\left(x_{1}+n-1\right)}{\Gamma\left(x_{1}+y_{2}+n-2\right)} x_{1}^{y_{2}-1} \Gamma\left(y_{2}+n-1\right) x_{1}^{1-y_{2}} \\
& =\sigma\left(x_{1}\right) .
\end{aligned}
$$

Applying (2.4) we get: if $y_{2}>1$, then $\lim _{x_{1} \rightarrow \infty} \sigma\left(x_{1}\right)=0$; and, if $y_{2} \in(0,1)$, then we have $\lim _{x_{1} \rightarrow \infty} \sigma\left(x_{1}\right)=\infty$. This implies that $\left(x_{1}, \ldots, x_{n}\right) \mapsto B\left(x_{1}, \ldots, x_{n}\right)$ is neither submultiplicative nor supermultiplicative on $\mathbf{R}_{+}^{n}$.

\section{References}

[1] M. Abramowitz and I. A. Stegun (eds.), Handbook of mathematical functions with formulas, graphs and mathematical tables (Dover, New York, 1965).

[2] H. Alzer, "On some inequalities for the gamma and psi functions", Math. Comp. 66 (1997) 373-389.

[3] H. Alzer, "Sharp inequalities for the beta function", Indag. Math. (N.S.) 12 (2001) 15-21.

[4] G. D. Anderson, M. K. Vamanmurthy and M. K. Vuorinen, Conformal invariants, inequalities, and quasiconformal maps (Wiley, New York, 1997). 
[5] G. E. Andrews, R. Askey and R. Roy, Special functions (Cambridge Univ. Press, Cambridge, 1999).

[6] E. F. Beckenbach, "Superadditivity inequalities", Pacific J. Math. 14 (1964) 421-438.

[7] A. M. Bruckner and E. Ostrow, "Some function classes related to the class of convex functions", Pacific J. Math. 12 (1962) 1203-1215.

[8] B. C. Carlson, Special functions of applied mathematics (Academic Press, New York, 1977).

[9] S. S. Dragomir, R. P. Agarwal and N. S. Barnett, "Inequalities for beta and gamma functions via some classical and new integral inequalities", J. Inequal. Appl. 5 (2000) 103-165.

[10] L. Gordon, "A stochastic approach to the gamma function", Amer. Math. Monthly 101 (1994) 858-865.

[11] E. Hille and R. S. Phillips, Functional analysis and semi-groups, Amer. Math. Soc. Coll. Publ. 31, revised ed. (AMS, Providence, RI, 1957).

[12] E. A. Karatsuba and M. Vuorinen, "On hypergeometric functions and generalizations of Legendre's relation", J. Math. Anal. Appl. 260 (2001) 623-640.

[13] G. Lochs, "Abschätzung spezieller Werte der unvollständigen Betafunktion", Anz. Österreich. Akad. Wiss. Math.-Natur. Kl. 123 (1986) 59-63.

[14] L. Maligranda, "Indices and interpolation", Dissertationes Math. (Rozprawy Mat.) 234 (1985) $1-54$.

[15] D. S. Mitrinovic, Analytic inequalities (Springer, New York, 1970).

[16] W. Raab, "Die Ungleichungen von Vietoris", Monatsh. Math. 98 (1984) 311-322.

[17] A. W. Roberts and D. E. Varberg, Convex functions (Academic Press, New York, 1973).

[18] R. A. Rosenbaum, "Sub-additive functions", Duke Math. J. 17 (1950) 227-247.

[19] L. Vietoris, "Über gewisse die unvollständige Betafunktion betreffende Ungleichungen", Sitzungsber. Österreich. Akad. Wiss. Math.-Natur. Kl. 191 (1982) 85-92. 